\section{Case Reports in Oncology}

\title{
Nivolumab-Induced Myocarditis Concomitant with Myasthenia Gravis
}

\author{
Yoko Fukasawa $^{\mathrm{a}}$ Kazuya Sasaki $^{\mathrm{b}}$ Maika Natsume $^{\mathrm{a}}$ \\ Makoto Nakashima ${ }^{b}$ Shuji Ota ${ }^{a}$ Kiyotaka Watanabe $^{a}$ \\ Yoshihisa Takahashic ${ }^{c}$ Fukuo Kondo ${ }^{d}$ Ken Kozuma $^{b}$ Nobuhiko Seki ${ }^{a}$ \\ ${ }^{a}$ Department of Medical Oncology, Teikyo University School of Medicine, Tokyo, Japan; \\ ${ }^{b}$ Department of Cardiology, Teikyo University Hospital, Tokyo, Japan; ' ${ }^{\text {Department of }}$ \\ Pathology, Graduate School of Medical Sciences, International University of Health and \\ Welfare, Narita, Japan; ${ }^{d}$ Department of Pathology, Teikyo University Hospital, \\ Tokyo, Japan
}

\section{Keywords}

Adenocarcinoma - Immunotherapy · Lung cancer · Nivolumab · Myocarditis · Myasthenia gravis $\cdot$ Herzmyasthenie $\cdot$ Anti-programmed cell death-1 monoclonal antibodies

\begin{abstract}
We report a 69-year-old female patient with advanced lung cancer who developed myocarditis concomitant with myasthenia gravis (MG), also known as "Herzmyasthenie," after 3 cycles of nivolumab administration. Her initial symptoms were general malaise and double vision. However, her myocarditis deteriorated rapidly the following day, necessitating a temporary pacemaker and noninvasive positive pressure ventilation in the intensive care unit. Immunohistochemical examination of a myocardial biopsy suggested an immune response on the basis of HLA associations. The patient also developed impaired adduction of her left eye and elevated serum levels of acetylcholine receptor antibody, suggesting the onset of MG. Her condition gradually improved after immediate methylprednisolone pulse therapy. This case of nivolumab-induced "Herzmyasthenie" highlights the need to be aware that fulminant myocarditis might occur at the same time as MG during treatment with anti-programmed cell death-1 monoclonal antibodies.




\section{Case Reports in Oncology}

\section{Introduction}

The treatment of patients with advanced lung cancer has been changed by the introduction of immune checkpoint inhibitors such as nivolumab and pembrolizumab, known as antiprogrammed cell death-1 monoclonal antibodies [1]. However, despite their clinical benefits, these antibodies may induce immune-related adverse events [2]. Here, we report on a patient with advanced lung cancer who developed fulminant myocarditis concurrent with myasthenia gravis (MG) following nivolumab administration.

\section{Case Report}

A 69-year-old female patient, Eastern Cooperative Oncology Group performance status 2 , with no history of autoimmune disorders, was treated with nivolumab as 3rd-line therapy for advanced lung adenocarcinoma with pleural dissemination and asymptomatic brain metastases. She complained of general malaise and double vision 1 week after her 3rd cycle of nivolumab administration and was subsequently admitted to our hospital because of progressive worsening of her symptoms.

On the 1st hospital day, diffuse ST segment elevation was found on electrocardiography, and her serum creatine kinase level was 1,156 U/L (normal range 41-153 U/L), with concurrent elevation of creatine kinase-MB isoenzyme and troponin I levels. She had no typical symptoms of angina pectoris, and ultrasound cardiography demonstrated good left ventricular ejection fraction with no segmental hypokynesis or pericardial effusion. Emergent coronary angiography and left ventriculography showed no abnormalities, thus excluding possible myocardial infarction. We therefore suspected immune-related myocarditis induced by nivolumab. After myocardial biopsy, pulse methylprednisolone was initiated at a dose of $1,000 \mathrm{mg}$ for 3 days followed by $1 \mathrm{mg} / \mathrm{kg} /$ day.

On the 2nd hospital day, the patient suddenly complained of dyspnea due to congestive heart failure, requiring temporary noninvasive positive pressure ventilation in the intensive care unit. She showed decreased blood pressure with complete atrioventricular block and multifocal premature ventricular contractions on electrocardiography, and a temporary cardiac pacemaker was therefore implanted simultaneously. She gradually improved under these supportive-care measures and continued steroid therapy, and the pacemaker was removed on the 7th hospital day.

Pathological examination of a myocardial biopsy demonstrated lymphocytic and neutrophilic infiltrations, interstitial edema, and myocardial necrosis. Moreover, immunohistochemistry demonstrated CD8 and CD4 T cells within the myocardial tissue, as well as expression of HLA-ABC and HLA-DR antigens on the myocardial cells, suggesting an immune response on the basis of HLA associations (Fig. 1). Serological and in situ analyses for cardiotropic viruses were negative.

Regarding the impaired adduction of her left eye, positivity for serum acetylcholine receptor antibody suggested MG. Furthermore, magnetic resonance imaging showed neither any new lesion in the brain nor the preexisting lesion in the medial longitudinal fasciculus. The patient was able to walk after rehabilitation training and continued steroid therapy, and left our hospital on the 36th hospital day. 


\section{Discussion}

Myocarditis concomitant with MG, or Herzmyasthenie, was first proposed as a new entity in 1901 [3]. The frequency of myocardial changes associated with MG has been reported to be as high as $16 \%(17 / 108)$ to $37 \%(28 / 75)[4,5]$. Some patients with MG have recently demonstrated autoimmune antibodies against heart or skeletal muscle as well as acetylcholine receptor, including antibodies against the 30-kDa titin fragment MGT-30, ryanodine receptor, and the voltage-gated potassium channel Kv1.4 [6]. Furthermore, several autoimmune-risk genes associated with $H L A$, including $A 1, B 8, D R 3, D R 14, D R 16$, and $D Q 5$, have been identified in myocardial biopsies from patients with MG, as in our case [7]. Myocardial changes associated with MG have mainly been found in patients with preexisting thymoma MG; however, our patient did not have thymoma, and the onset of myocarditis was simultaneous with the onset of MG [4-6]. This highlights the possibility that fulminant myocarditis might occur at the same time as non-thymoma MG during treatment with anti-programmed cell death-1 monoclonal antibodies.

Several cases of myocarditis associated with immune checkpoint blockade have been published [8,9], but to the best of our knowledge, the current case provides the first report of critical nivolumab-induced Herzmyasthenie in a patient with advanced lung cancer.

\section{Statement of Ethics}

The authors have no ethical conflicts to disclose.

\section{Disclosure Statement}

The authors declare that they have no relevant financial interests.

\section{References}

1 Morgensztern D, Herbst RS: Nivolumab and pembrolizumab for non-small cell lung cancer. Clin Cancer Res 2016;22:3713-3717.

-2 Zimmer L, Goldinger SM, Hofmann L, et al: Neurological, respiratory, musculoskeletal, cardiac and ocular side-effects of anti-PD-1 therapy. Eur J Cancer 2016;60:210-225.

3 Laquer L: Ueber die Erb'sche Krankheit (Myasthenia gravis). Neurolog Zentralblatt 1901;20:594-597.

-4 Hofstad H, Ohm OJ, Mørk SJ, Aarli JA: Heart disease in myasthenia gravis. Acta Neurol Scand 1984;70:176-184.

5 Gibson TC: The heart in myasthenia gravis. Am Heart J 1975;90:389-396.

-6 Suzuki S, Utsugisawa K, Yoshikawa H, et al: Autoimmune targets of heart and skeletal muscles in myasthenia gravis. Arch Neurol 2009;66:1334-1338.

7 Gilhus NE, Verschuuren JJ: Myasthenia gravis: subgroup classification and therapeutic strategies. Lancet Neurol 2015;14:1023-1036.

-8 Heinzerling L, Ott PA, Hodi FS, et al: Cardiotoxicity associated with CTLA4 and PD1 blocking immunotherapy. J Immunother Cancer 2016;4:50.

9 Johnson DB, Balko JM, Compton ML, et al: Fulminant myocarditis with combination immune checkpoint blockade. N Engl J Med 2016;375:1749-1755. 


\section{Case Reports in Oncology}
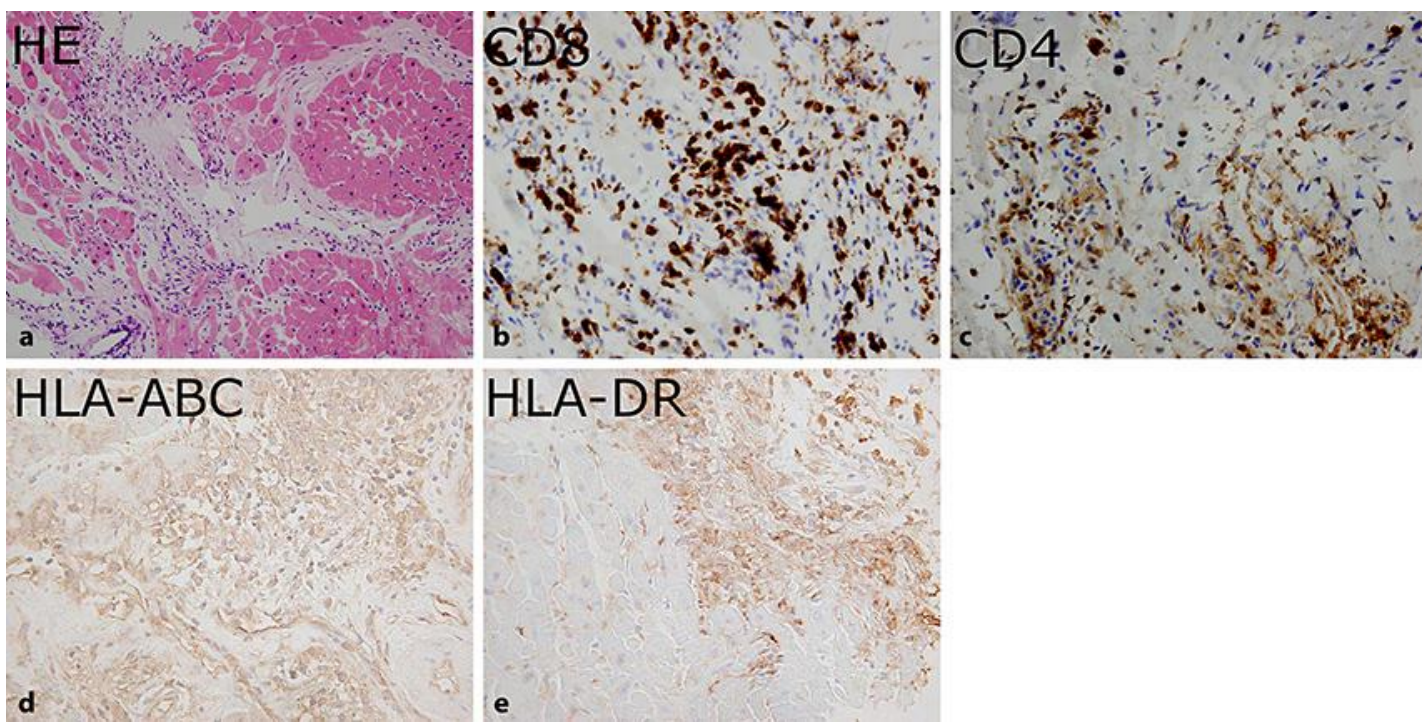

Fig. 1. Pathological findings of myocardial biopsy. a Myocardial tissue stained with hematoxylin and eosin (HE) demonstrated lymphocytic and neutrophilic infiltrations, interstitial edema, and myocardial necrosis. Immunohistochemical analysis demonstrated CD8 T cells (b) and CD4 T cells (c) within the myocardial tissue, as well as expression of HLA-ABC antigen (d) and HLA-DR antigen (e) on the myocardial cells. Original magnifications: $\mathbf{a} \times 20, \mathbf{b} \times 40, \mathrm{c} \times 40, \mathbf{d} \times 200$, e $\times 200$. 